\title{
EL PRESENTE DE SUBJUNTIVO EN ALGUNAS CLÁUSULAS SUBORDINADAS EN EL ESPAÑOL DE ARGENTINA
}

\begin{abstract}
Prymak Sergio Leonel, El presente de subjuntivo en algunas cláusulas subordinadas en el español de Argentina [The present of the subjunctive mood in some subordinated clauses of the Argentine Spanish]. Studia Romanica Posnaniensia, Adam Mickicwicz University Press, Poznań, vol. XXXI: 2004, pp. 437-441. ISBN 83-232-1353-4, ISSN 0137-2475.
\end{abstract}

The title of this work "The present of the subjunctive mood in some subordinated clauses of the Argentine Spanish" indicates a typical use for Argentine Spanish. Some authors mentioned this use as "dislocated use". Admitting this concept, the author introduces the concept "actualization of the message" as a systematic linguistic resource for these clauses. Finally, it is remarked that there is the possibility of extension of this use to other dialects.

Esta ponencia sobre el uso del presente de subjuntivo en el español de Argentina retoma aspectos ya mencionados anteriormente por Kany (1945), Lipsky (1996) Rojo y Veiga (1999) sobre el tema, y además de dar una explicación al valor temporal que adquiere el presente de subjuntivo en oraciones subordinadas, se plantea la cuestión de si este uso sistemático no lleva también a una actualización del discurso.

Hago referencia, por lo tanto, al presente de subjuntivo en las oraciones subordinadas con uso obligatorio, como causa del régimen modal del verbo en la principal (quiero que me digas la verdad). El vector temporal que forma el presente de subjuntivo en relación con el momento de la enunciación en la subordinada puede ser de simultaneidad (no creo que tenga razón) o posterioridad (te pido que compres el pan), no así de anterioridad. Aclaro, que en el caso de posterioridad en el ejemplo citado, no es una relación básica, ya que en el modo subjuntivo esta relación no se presenta. A este tipo de uso se lo ha llamado uso recto. En contraposición, cuando el vector que se forma es de anterioridad, se lo llama uso dislocado (Rojo y Veiga, 1999: 2911). Por eso, en las oraciones del tipo No quería que se vaya (sólo paradigmática, como los otros ejemplos hasta ahora) el presente 
de subjuntivo no aparece con un uso recto, sino dislocado. Sin embargo, también se puede encontrar el mismo tiempo y modo después de pretérito indefinido, por ejemplo, José me dijo que haga la comida (y todavía no la he hecho) no significando por ello un uso dislocado. Lo interesante del caso es que el presente de subjuntivo en algunas subordinadas alterna con el imperfecto de subjuntivo. Entonces, se nos plantean las siguientes preguntas: $i S i e m p r e$ aparece con el mismo valor de anterioridad? ¿Se puede decir que siempre su uso es dislocado? ¿Acaso no hay una actualización del mensaje? Si no es un uso dislocado por su intención y sus valores en ejemplos del tipo "José me dijo que venga", ¿puede que se generalice, a partir de este tipo de oraciones a otras variantes del español? Valga la aclaración de que este uso dislocado no es extensivo a todos los registros del español de Argentina, los ejemplos que se presentan en la ponencia son de la prensa, aunque no por ello, se pueda decir que son extensivos a todas las oraciones, como tampoco se puede afirmar que en todos los casos se haya dejado de usar el imperfecto de subjuntivo en la lengua hablada, aunque sí se ha podido constatar su uso en hablantes nativos argentinos, y en el mismo sentido de lo expuesto, no se puede afirmar que su uso sea sistematizado y en todos los casos. Aclarado esto, quiero presentar en cuatro puntos concisos el tema.

1. Un uso dislocado. No hay duda de que en algunas de estas oraciones el uso del presente de subjuntivo no es dislocado, sino recto. Igual, muchos autores ponen en duda el valor temporal exacto de los tiempos de subjuntivo Borrego et. alias (1986), Suñer (1990), Suñer y Padilla Rivera (1990), Porto Dapena (1991), AndresSuárez (1994), Sastre (1997) Siguiendo la definición de dislocación temporal de Rojo y Veiga (op. cit., 2911) ${ }^{1}$, no parece haber problemas con el reconocimiento del siguiente ejemplo, donde se emplea con un uso recto: "El juez hizo lugar a una acción de amparo interpuesta por la escritora y actriz María Cristina Bosch, quien consiguió así que para su caso particular se deje sin efecto las restricciones que fijó el Gobiemo para los retiros de dinero depositado en bancos y entidades financieras" (Clarín, 14.12.2001). Como vemos en esta oración el verbo de la principal hace referencia al pasado y el de la subordinada en presente de subjuntivo al futuro con relación al momento de enunciación del mensaje. Ahora bien, en este otro ejemplo, "Estaba cansado de que no me paguen, de que gente que me hizo daño siga en el fútbol, de ser utilizado, de que te exijan demás..." (Clarín, 8.09.2001), se ve con claridad que en el uso del verbo en la subordinada pagar hace referencia a anterioridad con relación al momento de la enunciación del mensaje, aunque no es seguro que suceda lo mismo con el verbo seguir, ya que su referencia temporal es

\footnotetext{
' Muchos autores coinciden en que no se puede clasificar con precisión los límites temporales de los tiempos de subjuntivo. Así Rojo y Veiga hablan de usos rectos y dislocados, señalando que "No todas las distinciones temporales que el funcionamiento del sistema verbal permitc entre unidades indicativas empleadas conforme a sus valores temporales rectos sobreviven en subjuntivo, siendo especialmente perceptible la inexistencia de formas específicas en este segundo modo para expresar relaciones básicas en que intervenga un vector de postcriotidad".
} 
hacer. Pero en el siguiente ejemplo ya no hay dudas de la dislocación temporal: "En el 98 le recomendó a su familia que reduzca gastos porque tenía sus ahorros en bonos y acciones" (Clarín, 23.12.2001). Queda claro que el verbo de la subordinada reducir hace referencia a un momento anterior a la enunciación del mensaje.

2. Equiparación del valor temporal con el imperfecto de subjuntivo. Muchas veces hay una equiparación del valor de anterioridad que denota el presente de subjuntivo, ya que aparece junto al imperfecto de subjuntivo en oraciones donde solo tendría que aparecer éste último. Por ejemplo: "Lo que pasa es que había miedo a que empataran y que llegue algún gol en el final. Eso es jodido (Maradona)" (Clarín, 9.10.2000). Es justamente en este tipo de oraciones donde se ve que el presente de subjuntivo adquiere un valor temporal que es equiparable al del imperfecto de subjuntivo. Esta alternancia de los dos tiempos demuestra este hecho.

3. Necesidades del hablante. Al utilizar el presente de subjuntivo el hablante presenta unas necesidades de actualización del mensaje. Puede que haya diferentes motivos para realizar esto. Existen otros casos en el español de actualización del mensaje, el problema está en que no siempre tiene que ser por necesidades, porque este uso del presente de subjuntivo se presenta más bien como sistemático, adquiriendo un uso en el sistema temporal del subjuntivo en la variante de Argentina que reemplaza al imperfecto de subjuntivo. Si esto es así, el propio sistema temporal está proporcionando una solución sistemática a esta actualización, usando el presente de subjuntivo para llevarla adelante. En la historia de otras lenguas o en la propia lengua española hay casos de cambio de un valor temporal (léase el cambio del pluscuamperfecto a imperfecto de subjuntivo con la forma -ra) o de adquisición de un valor o uso secundario por parte de un tiempo (léase el futuro simple con valor hipotético). En mi opinión, el sistema le ha dado al hablante una solución al problema de actualización del mensaje, dándole al presente de subjuntivo un valor de anterioridad que no tiene en otras variantes, y por consiguiente, tiene un uso de actualizador en este tipo de oraciones. Entiendo que la actualización del discurso es un recurso linguístico que se realiza también con otros tiempos del modo indicativo en español, y no es casualidad que los ejemplos sean de la prensa donde la actualización se utiliza como recurso lingüístico narrativo con mucha frecuencia, y siempre, en los ejemplos de la lengua hablada donde se ha encontrado este recurso, ha formado parte de conversaciones donde interesaba traer ese elemento al presente ("no quería que se vaya", hablando de una persona que se había ido recientemente).

4. El porqué. Se pueden encontrar múltiples hipótesis para este uso. Aquí se presenta la actualización, pero no por ello se pueden dejar de lado otras ${ }^{2}$. Quedan

${ }^{2}$ G. Rojo (1976) saca la conclusión que "Las construcciones del tipo Les pedí que lo hagan expresando una relación $\mathrm{O}+\mathrm{V}$ ('futuro') independiente de la represcntada por el verbo principal, $\mathrm{O}-\mathrm{V}$ ("pretérito"), podrian constituir el punto de partida de una tendencia a usar cante para expresar, en general, posterioridad a un 'pretérito' aun cuando el proceso expresado por el verbo dependiente no fuese simultáneo ni posterior al origen, lo que supondría el deslizamiento de cante al dominio de las relaciones temporales con vector originario de anterioridad". 
dos cuestiones sin resolver. Una, por qué elemento de la oración se introduce este uso y dos, si es un una tendencia o proceso que no tiene vuelta atrás. Es claro que se puede hablar de fenómeno extensivo. Por un lado, en muchas variantes del español, el uso del pretérito indefinido ha relegado a un segundo plano al uso del pretérito perfecto. Por otro lado, al usarse el indefinido, la correlación temporal normativa "Me ha dicho que venga", deja de corresponderse, y por eso, en mi opinión, es justamente por este desplazamiento de los verbos de la principal que se puede hacer extensivo el uso del presente de subjuntivo en la subordinada a otras variantes del español.

Todo cambio conlleva en un sentido cierta innovación, lo cual no exige que el cambio provenga necesariamente de elementos que estén fuera del sistema. Y es justamente lo que se presenta aquí. Una cita de W. Nowikow (1995: 30) lo puede aclarar mejor: "Las innovaciones se imponen como actos sociales, haciéndolo no obstante, a partir de ciertos elementos que forman parte del sistema, y respondiendo a ciertas posibilidades y necesidades sistemáticas". Si es cierto esto, no se puede saber si este cambio, que ya lleva muchos años en el habla y la escritura de la variante argentina, se generalizará a otras variantes o retrocederá y quedará como algo anecdótico. No todos los cambios en la lengua terminan plasmándose de forma clara, y mucho menos, se hacen extensivos a todo un continente tan rápidamente. El primer libro de lingüística que data este registro es de 1945 (Kany). Asegurar que en esa fecha se lo dató, no significa que el uso no se remonte a otras variantes del español, y que su uso no existiera antes de la misma. Por eso, puede que estemos ante un fenómeno de reducción general de los tiempos de subjuntivo como en el francés, aunque para constatar esta suposición habría que hacer un estudio mucho más amplio que cae fuera de esta comunicación.

\section{REFERENCIAS BIBLIOGRÁFICAS}

LIBROS

Andres-Suárez, I. (1994), El verbo español - sistemas medievales y sistema clásico. Madrid: Gredos.

Borrego, J., A sencio, J.G., Prieto, E. (1986), El subjuntivo - valores y usos. Madrid: Sociedad Española de Librerías, S.A.

Lipsk y, J. M. (1996), El español de América. Madrid: Cátedra.

Porto Dapena, J. Á. (1991), Del indicativo al subjuntivo - valores y usos de los modos del verbo. Madrid: Arco/Libro S.A.

Sastre, M. Á. (1997), El subjuntivo en español. Salamanca: Ediciones Colegio de España.

LIBROS TRADUCIDOS

Kany, Ch. E. (1945), Sintaxis hispanoamericana, trad. Martín Blanco Álvarez. Madrid: Gredos, 1994. 


\section{COLABORACIÓN EN LIBRO COLECTIVO}

Rojo, G., Veiga, A. (1999), El tiempo verbal. Los tiempos simples, en Gramática descriptiva de la lengua española, Tomo dos, I. Bosque, V. Dernonte (eds). Madrid: Espasa, 2867-2934.

Suñer, M. (1990), El tiempo en las subordinadas, en Tiempo y Aspecto en español, I. Bosque (ed.). Madrid: Cátedra, 77-105.

Suñer, M., Padilla Rivera, J. (1990), Concordancia temporal y subjuntivo, en Indicativo y Subjuntivo, I. Bosque (ed.). Madrid: Taurus Ediciones, 185-201.

\section{ARTÍCULOS Y COMUNICACIONES}

Nowikow, W. (1995), La causación múltiple y la polinterpretación del cambio lingǘstico, Acta Universitatis Wratislaviensis Núm. 160. Wrocław: Wydawnictwo Unywersytetu Wroclawskiego, 29-39.

Rojo, G. (1976), La correlación temporal, Verba 3, 65-68. 